\title{
Subject Index to Volume 18
}

Acetaminophen, influenza B virus and, Reye's syndrome, mouse, 181

Acetyl-CoA: $\alpha$-glucosamine $N$-acetyltransferase, Sanfilippo syndrome, diagnosis with, 543

Acidemia

isovaleric, isovaleryl glucuronide excretion patterns, 508

propionic and methylmalonic, dicarboxylic acid identification, amniotic fluid and urine, 1185

Acidosis

cerebral blood flow and, fetal lamb, 1309

lactic

fibroblast defect correction, neonate, 1144

respiratory complex III component deficiency, 991

respiratory, cardiac output distribution, fetal and neonatal lamb, 731

Action potential, developmental changes, ventricular conducting system, dog, 53

Acylcarnitine, urinary excretion, organic acid metabolism disorders, 1325

Adenine phosphoribosyltransferase, complete deficiency, 30

Adenosine, analogue, ventilation depression, neonatal rabbit, 387

Adenosine $3^{\prime}, 5^{\prime}$-monophosphate, human chorionic gonadotropin-stimulated production, zinc ion effects, testis, rat, 232

Adenylate cyclase, insulin and glucagon receptors, ontogeny, liver, neonatal guinea pig, 558

Adolescence, blood minerals, circadian rhythms, 456

Adrenoleukodystrophy, 286

$\beta$-Adrenoreceptor, alveolar, glucocorticoid effects, fetal rabbit, 1191

Agar, intestinal secretion, bilirubin-induced, reversal by, hamster, 79

Agar bead, Pseudomonas aeruginosa, pathogenicity, role in, respiratory tract, rat, 295

Age, maternal, Down's syndrome and, mathematical function, 375

Airway

function, lung distensibility and, asthma, 1154

hyperreactivity, cold air challenge, 469

upper, muscle regulation, progressive asphyxia, rabbit, 819

Albumin, iodinated compounds and thyroxine binding to, human milk, 901

Aldosterone, secretion, factors controlling, hypoxemia, fetal lamb, 607

Alloantigen, lymphocyte response to, neonate, 414

Alloxan, diabetes, myocardial excitationcontraction coupling, fetal rabbit, 1344

Alveolus

$\beta$-adrenoreceptor, glucocorticoid effects, fetal rabbit, 1191

epithelial solute permeability, surfactant deficiency, lung, fetal lamb, 566

partial carbon dioxide pressure, variations, brain blood flow and cardiac output, neonatal piglet, 1132
Amenorrhea, hypothalamic, opiate antagonism and gonadotropin secretion, 322

American Academy of Pediatrics, Pediatric Section Scientific Meeting, 306

Amino acid, sulfur, altered intake, brush border membrane response, rat, 611

Amniocyte, very long chain fatty acids, impaired oxidation, adrenoleukodystrophy, 286

Amniotic fluid

dicarboxylic acid, identification, propionic and methylmalonic acidemia, 1185

galactitol, analysis, prenatal galactosemia diagnosis, 714

$\gamma$-glutamyl transpeptidase deficiency, correction, cystic fibrosis, fetus, 1340

immunoreactive trypsin, cystic fibrosis, fetus, 957

vasopressin concentration, hypoxia index, fetal sheep, 552,835

Androgen

receptor, binding abnormality, hypospadias, neonate, 216

therapy, height and, 467

Anemia

chronic, gastrointestinal blood flow and oxygen consumption, effects on, neonatal lamb, 420

hemolytic poikilocytic, spectrin defect, 1005

Anion

exchange mechanism, abnormal, sweat glands, cystic fibrosis, letter, 496

renal, transport, Mendelian mutation and, human and mouse, 25

transport, 2,3-diphosphoglycerate and, cystic fibrosis erythrocytes, 1017

Antibiotics, Escherichia coli $\mathrm{K} 1$, fulminant sepsis, neonatal rabbit, 314

Antibody

antiviral, human, herpes simplex virus infection from, protection ontogeny, neonatal mouse, 1164

hybridoma, group B streptococcal infection, treatment with, neonatal rat, 1093

IgG, carbohydrate and protein antigens, group B streptococci, mouse, 478

ingested antigen, altered response, enterocolitis, 751

monoclonal

2-2-B, Escherichia coli K1-killing, mouse, 770

cystic fibrosis $\alpha_{2}$-macroglobulin, 999

pneumococcal polysaccharide, responses to, neonate, 1067

Pseudomonas aeruginosa mucoid exopolysaccharide and sodium alginate, cystic fibrosis serum, 431

respiratory syncytial virus, 987

Antigen

carbohydrate and protein, IgG antibodies to, group B streptococci, mouse, 478

human lymphocyte, DR type, diabetes manifestations and, 1239

ingested, altered antibody response, enterocolitis, 751

protein, immune response, mouse, 588

Antithrombin III, low levels, idiopathic res- piratory distress syndrome prognosis and, neonate, 273

Apoliprotein, Menkes' disease, neonate, 864

Arachidonic acid, thromboxane production stimulation, platelets, neonatal and adult, 823

Arginine vasopressin, blood pressure control, rat, 701

Artery, pulmonary and femoral, $l$-norepinephrine stimulation, neonatal lamb, 244

Asphyxia

birth, thromboxane $B_{2}$ production, neonate and fetus, 756

myocardial blood flow during, regional distribution and determinants, fetal lamb, 764

progressive, upper airway muscle regulation, rabbit, 819

Asthma, lung distensibility, airway function and, 1154

Atrium, pacing, tachycardia induced by, cardiac function and myocardial metabolism during, dog, 369

Atropine, cerebral blood flow, effects on, hypertension, neonatal piglet, 1121

Auditory brainstem evoked response, fetal lamb, 83

Auditory brainstem response, current testing, neonate, 780

Bacteremia, group B streptococcal, tolazoline in, neonatal piglet, 960

Bacteria

adherence

intravenous catheters and needles, phlebitis and, rabbit, 1361

postnatal age and, intestine, guinea pig, 1366

Barnett, Henry Louis

Howland Award, 1379

acceptance, 1383

Behavior

early malnutrition and

learning disabilities, 309

soft neurologic signs, 826

Benn index, weight and height accretion, relative velocity, 627

Betamethasone, L-carnitine vs., dipalmitoylphosphtidylcholine content, lung, fetal rat, 1246

Bile salt, lipase and esterase activity stimulated by, collected, stored, and heated human milk, 382

Bilirubin

binding, plasma, fluorescence quenching vs. peroxidase, neonate, 349

DNA damage, phototherapy effects, human and puppy, 3

intestinal secretion induced by, reversal by agar, hamster, 79

photo-derivatives, water solubility of, $\mathrm{pH}$ dependence, phototherapy and, 378

photooxidation products, urine, jaundice, neonate, 696

serum concentrations, jaundice, neonate, letter, 682

Biopsy, trophoblast, lysosomal disease diagnosis, first trimester, 1032 
Birth

asphyxia, thromboxane $\mathrm{B}_{2}$ production, neonate and fetus, 756

onset of breathing, pulmonary vascular prostacyclin synthesis, lamb and goat, 938

Birth weight

low, longitudinal metabolic studies, 254

very low, intrauterine growth retardation, neonate, 709

Blockade, calcium channel, heart contractile response, effects on, neonatal and adult rabbit, 948

Blood

citrated, exchange transfusions, plasma vitamin $\mathrm{D}$ and, neonate, 429

minerals, circadian rhythms, adolescence, 456

substrate-oxygen levels, uterus, pregnant guinea pig, 1172

Blood flow

cerebral

atropine effects, hypertension, neonatal piglet, 1121

autoregulation, arterial oxygenation and, fetal lamb, letter, 572

electrical impedance vs. strain gauge plethysmography, neonate, 290

hypoxia, hypercapnia, acidosis, and hypotension, fetal lamb, 1309

intraventricular hemorrhage and, puppy, 7

$\mathrm{PaCO}_{2}$ variations, neonatal piglet, 1132

pancuronium bromide and, neonatal lamb, 1305

distribution, nitroprusside effects, neonatal lamb, 618

gastrointestinal, anemia and hypoxia effects on, neonatal lamb, 420

myocardial

increased, hypoxemia, neonatal lamb, 602

regional distribution and determinants, asphyxia, fetal lamb, 764

placental, decreased, diabetes and, rat, 735

renal, response to hemorrhage, fetal lamb, 40

retinal and choroidal, hypoxic and hypercapnic neonatal lamb, 410

Blood pressure, control, arginine vasopressin, rat, 701

Blood vessel

prostaglandin biosynthetic activities, fetal lamb, 12

pulmonary

prostacyclin synthesis, onset of breathing at birth, lamb and goat, 938

resistance, meclofenamate effects on, piglet, 579

pulmonary and peripheral, short-term responses, group B streptococcus, oophorectomy and, sheep, 266

Body composition, total parenteral nutrition fuel mixes and, neonatal miniature pig, 261

Body water, measurement, $\mathrm{H}_{2}{ }^{18} \mathrm{O}$ isotopic determinations, neonate, 524

Brain

blood flow, see Blood flow, cerebral

development, diabetes, fetal guinea pig, 650

malnourished, theophylline and, neonatal rat, 546

maternal corticosteroid therapy and, fetal monkey, 440

metabolic fuel, lactate, glucose-6-phosphatase deficiency and, 335

ornithine carbamoyltransferase, defi- ciency, tryptophan and 5-hydroxyindole acetic acid increase, brain, mouse, 372

pyruvate metabolism, dichloroacetate effects, rat, 936

Breast feeding

see also Human milk

bottle vs., fatness and serum cholesterol indices, 8-year-old, 1233

Bromide, distribution volume, letter, 392

Bronchopulmonary dysplasia, pathogenic factors, 483

Brush border membrane, sulfur amino acids, altered intake, rat, 611

\section{Calcium}

metabolism, mitochondrial abnormalities, cystic fibrosis and, 594

parenteral treatment, left ventricular systolic time intervals and, hypocalcemia, neonate, 71

total, 25-hydroxyvitamin D and, concentrations, Saudi mothers and neonates, 739

transport, intestinal, kinetics during maturation, rat, 235

Calcium antagonist, hypoxic pulmonary hypertension, piglet, 1262

Calcium-ATPase, erythrocyte membranes, activity in, cystic fibrosis, 890

Calcium channel blocker, verapamil, nifedipine, and diltiazem, heart contractile response, effects on, neonatal and adult rabbit, 948

Carbohydrate

antigens, IgG antibodies to, group B streptococci, mouse, 478

composition, monkey milk, 911

slow-release, type I glycogen storage disease, 879

tolerance, pancreatic exocrine function and, cystic fibrosis, 1107

Carbon dioxide, partial alveolar pressure, variations, brain blood flow and cardiac output, neonatal piglet, 1132

Carbonic acid, dissociation constant, critically ill neonate, 1287

Cardiomyopathy, histiocytoid, neonate, 1023

Cardiopulmonary system

adaptations of transition, indomethacin and, rabbit, 842

measurement, rebreathing methodology, piglet, 1167

Cardiovascular system

group B streptococcal sepsis, changes in, piglet, 874

hypocapnic effects, puppy, 685

hypoxemia, responses to, chemical sympathectomy, fetal lamb, 318

Carnitine

protective effect, diphtheric myocarditis, 815

tissue reserves, neonate, 679

L-Carnitine

betamethasone vs., dipalmitoylphosphatidylcholine content, lung, fetal rat, 1246

urinary excretion, organic acid metabolism disorders, 1325

Cartilage, costal, reduced sulfate uptake, growth retardation, fetal rat, 1100

Casein

methylated, anticholeraic effect, jejunum, rat, 1075

whey and, different proportions, nitrogen metabolism, neonatal pig, 658

Catecholamine, hypoxemia, responses to, chemical sympathectomy, fetal lamb, 318

Catheter, intravenous, bacterial adherence to, phlebitis and, rabbit, 1361

Catheterization, chronic, glucose turnover rates, pregnancy and, rabbit, 276

Cation, monovalent, transport, erythrocytes, neonate and adult, 778

Celiac disease, cereal components toxic in, intestine, fetal rat, 1372

Cereal peptide, toxicity, celiac disease, intestine, fetal rat, 1372

$\left[{ }^{14} \mathrm{C}\right]$ Glucosamine, substrate, acetyl-CoA: $\alpha$ glucosamine $N$-acetyltransferase assay, Sanfilippo syndrome, 543

Chemotaxis, polymorphonuclear leukocyte and monocyte inhibition, hyperimmunoglobulin-E-associated infection, 365

Chloral hydrate, genioglossus and diaphragmatic activity, effect on, 516

Cholera toxin

binding, microvillus membrane differentiation, intestine, newborn and adult rabbits, 984

methylated casein, effects on, jejunum, rat, 1075

Cholestasis, tryptophan, neonatal rat, 904

Cholesterol, serum, index, neonatal feeding and growth, correlation between, 1233

Cholesterol ester storage disease, cholesterol ester and triglyceride metabolism, 1242

Cholinephosphotransferase, developmental changes, lung, fetal and neonatal rabbit, 395

Choroidea, blood flow, see Blood flow, choroidal

Cilia, dyskinetic activity, oligosaccharide component and, cystic fibrosis, 540

Cimetidine, long-term, cystic fibrosis, 66

Circadian rhythm, blood minerals, adolescence, 456

Clinical Research Centers, perinatology advances, 197

Cold air challenge, airway hyperreactivity, 469

Collagen, type I/III, elevated ratios, chronic ventilation, respiratory distress syndrome, neonate, 1176

Colostrum, enteric mucosa growth induced by, puppy, 512

Complement

activation, phagocyte adherence reduction, 637

pathway, classical and alternative, preterm and small for gestational age neonate, 281

Cord blood

granulocytes and monocytes, 1127

mononuclear leukocytes, membrane fluid properties, increased insulin receptors and, 773

neutrophils

monoclonal antibody 2-2-B and, Escherichia coli $\mathrm{K} 1$, effects on, mouse, 770 oxidative metabolism, cytoplasmic granules and, neonate, 1148

Corticosteroid, hyaline membrane disease, maternal therapy, fetal brain and, monkey, 440

Cortisol, fetal/maternal water transfer, effects on, lamb, 631

Cyclic AMP, see Adenosine $3^{\prime}, 5^{\prime}$-monophosphate

Cysteine, total parenteral nutrition, supplement to, puppy, 747 
Cystic fibrosis

amniotic fluid, immunoreactive trypsin in, fetus, 957

anion exchange mechanism, abnormal, sweat glands, letter, 496

calcium-ATPase activity, erythrocyte membranes, 890

calcium metabolism and, mitochondrial abnormalities, 594

carbohydrate tolerance, pancreatic exocrine function and, 1107

ciliary dyskinetic activity, oligosaccharide component and, 540

cimetidine, long-term, 66

erythrocyte membrane phospholipids, abnormal fatty acid turnover, 704

erythrocytes, anion transport and 2,3-diphosphoglycerate in, 1017

erythrocytes and peripheral blood lymphocytes, $\mathrm{Cl}^{-}$permeabilities, 1336

$\gamma$-glutamyl transpeptidase deficiency, amniotic fluid, correction, fetus, 1340

lymphocytes, methylprednisolone resistance of, 488

$\alpha_{2}$-macroglobulin, monoclonal antibodies and, 999

protein detection, isoelectric focusing of serum and plasma, 130

Pseudomonas aeruginosa mucoid exopolysaccharide and sodium alginate, antibody to, 431

reserpine and isoproterenol, pulmonary function, rat, 1029

sweat factor, apparent absence, 1292

Cytidine $5^{\prime}$-diphosphate reductase, phytohemagglutinin-stimulated lymphocytes, immunodeficiency and, 691

Cytidylyltransferase, developmental changes, lung, fetal and neonatal rabbit, 395

Cytochrome $b$, reducible, deficiency, histiocytoid cardiomyopathy, neonate, 1023

2-Deoxyglucose, fasting and, hypoglycemia detection, 359,490

Deoxyribonucleic acid

damage, phototherapy-induced, bilirubin enhancement, 3

synthesis, weaning, diet composition, and glucocorticoid effects on, intestinal development, suckling rat, 145

Dexamethasone, prenatal, immunoreactive 6-ketoprostaglandin $F_{1 \alpha}$ levels, lung, fetal rat, 908

Diabetes

alloxan, myocardial excitation-contraction coupling, fetal rabbit, 1344

early clinical manifestations, human lymphocyte antigen DR types and, 1239

fetal brain development, guinea pig, 650

insulin-dependent, streptozotocin-induced, pertussis virus effects, mouse, 221

maternal, glycosylated hemoglobin and plasma protein, neonate, 767

pregnancy and, decreased placental blood flow, rat, 735

Dialysis, continuous ambulatory peritoneal, renal osteodystrophy and, 742

Diamine oxidase, disaccharidase and, small intestinal biopsies, 647

Diaphragm, activity, genioglossus and, chloral hydrate, 516

Dicarboxylic acid, methyl-branched chain identification in amniotic fluid and urine, propionic and methylmalonic acidemia, 1185
Dichloroacetate, pyruvate metabolism and, brain, rat, 936

Diet

glycogen storage disease type I, slow release carbohydrate, treatment with, 879

high galactose, male gonad resistance to, rat, 345

thymidine kinase activity and DNA synthesis, effects on, intestinal development, suckling rat, 145

Digesta, composition, nitrogen metabolism and, casein and whey proportions, neonatal pig, 658

Digestion

fat

lingual lipase, stomach and upper small intestine, rat, 402

lingual lipase stability, stomach, rat, 248

Digoxin, immunoreactive substance similar to, perinatal changes, neonate, 1097

$5 \alpha$-Dihydrotestosterone, receptor binding, abnormality, hypospadias, neonate, 216

Diltiazem, heart contractile response, effects on, neonatal and adult rabbit, 948

Dipalmitoylphosphatidylcholine, betamethasone vs. L-carnitine effects, lung, fetal rat, 1246

2,3-Diphosphoglycerate, anion transport and, cystic fibrosis erythrocytes, 1017

Diphtheria, myocarditis, carnitine protective effect, 815

Disaccharidase, diamine oxidase and, small intestinal biopsies, 647

DNA, see Deoxyribonucleic acid

Down's syndrome

blood platelets, decreased calcium content and ${ }^{45} \mathrm{Ca}^{2+}$ uptake, 914

maternal age and, mathematical function, 375

Ductus arteriosus

patency, respiratory distress and, recovery, prostaglandin excretion rates, premature neonate, 520

patency and closure, prostaglandin biosynthetic and catabolic enzymes, fetal lamb, 19

prostaglandin biosynthetic activities, fetal lamb, 12

Dysplasia, bronchopulmonary, pathogenic factors, 483

Dysrhythmia, ventricular, heart surgery and, dog, 1112

Electrical impedance, strain gauge plethysmography vs., cerebral blood flow study, neonate, 290

Electron transfer flavoprotein, defect, glutaric aciduria type II, neonate, 663

Electrophoresis, pseudo-arylsulfatase A deficiency, diagnosis with, 1042

Endocrinology, malnutrition and, letter, 300

Endothelium, umbilical vein, maternal smoking effects, 1071

Energy cost, growth, low birth weight and, neonate, 254

Enteric mucosa, colostrum-induced growth, puppy, 512

Enterocolitis, food protein-induced, antibody response to ingested antigen, 751

Enzyme

antioxidant

birth into oxygen-rich environment, lung, rabbit, 240 pulmonary, maturation, fetal and neonatal rat, 584,871

defect, tyrosinemia type I, 463

human milk, stability, gastrointestinal tract, 532

lung, developmental changes, fetal and neonatal rabbit, 395

prostaglandin, biosynthetic and catabolic, ductus arteriosus patency and closure, fetal lamb, 19

Epidermal growth factor, urine, thyroxine effects, neonatal mouse, 1080

Epinephrine, thromboxane production stimulation, platelets, neonate and adult, 823

Errata, 226, 495, 565, 683, 788

Erythrocyte

aggregation, premature and term, neonate and adult, 1356

$\mathrm{Cl}^{-}$permeabilities, cystic fibrosis, 1336

cystic fibrosis

anion transport and 2,3-diphosphoglycerate in, 1017

calcium-ATPase activity in, 890

fatty acids, protein-calorie malnutrition, 162

membrane, freeze-etching and biochemical analysis, fetus, 1035

membrane phospholipid, abnormal fatty acid turnover, cystic fibrosis, human, 704

monovalent cation transport, neonate and adult, 778

Erythrocyte 2,3-diphosphoglycerate, $\mathrm{Po}_{2} 50 \%$, and available oxygen, postnatal fall in hemoglobin, rabbit, 154

Erythropoiesis, fetal, reactivation, postnatal period, 178

Escherichia coli, 0124 and 0128 , adherence, postnatal age and, intestine, guinea pig, 1366

Escherichia coli $\mathrm{K} 1$

monoclonal antibody 2-2-B killing, mouse, 770

sepsis, fulminant, antibiotic therapy, neonatal rabbit, 314

Esterase, bile salt-stimulated activity, collected, stored, and heated human milk, 382

Eupnea, maturation, neonatal monkey, 943

European Society for Paediatric Endocrinology, annual meeting abstracts, 101,1206

European Society for Paediatric Gastroenterology and Nutrition, annual meeting abstracts, 1048

European Society for Paediatric Research, Working Group on Mineral Metabolism, and Paediatric Pathology Society, annual meeting abstracts, 795

European Society for Paediatric Research and Working Group for Mineral Metabolism, annual meeting abstracts, 86

Exercise

progressive

$\alpha$-ketoacids and, maple syrup urine disease, neonate, 851

oxygen uptake and heart rate, growthrelated changes, 845

Exopolysaccharide, mucoid, Pseudomonas aeruginosa, antibody to, cystic fibrosis serum, 431

Fasting

2-deoxyglucose test and, hypoglycemia detection, 359, 490

splanchnic uptake and energy substrate 
release, neonatal baboon, 1316

Fat

composition, monkey milk, 911

digestion

lingual lipase, stomach and upper small intestine, rat, 402

lingual lipase stability, stomach, rat, 248

Fatness, index, neonatal feeding and growth, correlation between, 1233

Fatty acid

abnormal turnover, erythrocyte membrane phospholipid, cystic fibrosis, 704

erythrocyte, protein-calorie malnutrition, 162

essential, deficiency, intravenous lipid emulsions, neonatal pig, 1350

free, arterial and venous concentrations, pregnant guinea pig, 1172

ketone bodies and, intestinal glucose metabolism, development, rat, 575

oxidation and ketogenesis, hepatic, neonatal guinea pig, letter, 299

very long chain, impaired oxidation in leukocytes, cultured skin fibroblasts, and amniocytes, adrenoleukodystrophy, 286

Ferritin, skeletal muscle, vitamin $\mathrm{E}$ defi-

Fetus ciency and, rabbit, 1012

alveolar $\beta$-adrenoreceptors, glucocorticoid effects, rabbit, 119

amniotic fluid

immunoreactive trypsin in, cystic fibrosis, 957

vasopressin in, hypoxia index, 552,835

asphyxia, myocardial blood flow, distribution and determinants, lamb, 764

auditory brainstem evoked response, lamb, 83

brain

development, diabetes, guinea pig, 650

blood flow, arterial oxygenation and, lamb, letter, 572

blood flow, hypoxia, hypercapnia, acidosis, and hypotension, lamb, 1309

maternal corticosteroid therapy and, monkey, 440

celiac disease, cereal component toxicity, intestine, rat, 1372

development, diabetes, decreased placental blood flow and, rat, 735

dipalmitoylphosphatidylcholine content and phosphatidylcholine species composition, lung, betamethasone vs. L-carnitine effects, rat, 1246

erythrocyte membranes, freeze-etching and biochemical analysis, 1035

erythropoiesis, reactivation, postnatal period, 178

glucocorticoid-thyroid hormone interactions, lung, rat, 191

$\gamma$-glutamyl transpeptidase deficiency, correction, cystic fibrosis, 1340

hemorrhage, renal response to, lamb, 40

hypoxemia, aldosterone secretion, factors controlling, lamb, 602

hypoxemia after chemical sympathectomy, cardiovascular and catecholamine responses, lamb, 318

hypoxia, vasopressin concentration in amniotic fluid, lamb, 552, 835

intra-amniotic triiodothyronine, pulmonary maturation, effects on, lamb, 932

lung liquid absorption and alveolar epithelial solute permeability, surfac- tant deficiency, lamb, 566

maternal smoking

growth retardation, rat, 127

xenobiotic metabolism, placenta and

umbilical vein endothelium, 1071

mother and, neurohypophyseal hormone

release, hypoxia effects on, sheep, 188

myocardial excitation-contraction cou-

pling, alloxan-diabetic, rabbit, 1344

neonate and

hypoxia, vasopressin excretion, lamb, 227

lung enzyme activities, developmental changes, rabbit, 395

pulmonary antioxidant enzyme maturation, rat, 584,871

respiratory acidosis, cardiac output distribution, lamb, 731

thromboxane $B_{2}$ production, respiratory distress syndrome and birth asphyxia, 756

prenatal dexamethasone, 6-ketoprostaglandin $\mathrm{F}_{1 \alpha}$ levels, lung, rat, 908

prostaglandin biosynthetic activities, ductus arteriosus, blood vessels, and lung tissue, lamb, 12

prostaglandin biosynthetic and catabolic enzymes, ductus arteriosus patency and closure, lamb, 19

pro-sucrase-isomaltase, high molecular weight, intestine, letter, 391

water transfer from mother, cortisol effects, lamb, 631

Fibroblast

cholesterol ester and triglyceride metabolism in, Wolman's disease and cholesterol ester storage disease, 1242

defect correction, lactic acidosis, neonate, 1144

$\beta$-galactosidase-neuraminidase deficiency, drug correction, 167

genital skin, androgen receptor binding, neonate, 216

Niemann-Pick disease types A, B, and C, sphingomyelinase activity in, 1088

skin

trace metal metabolism, mouse, 1282

very long chain fatty acids, impaired oxidation, adrenoleukodystrophy, 286

Flavoprotein, electron transfer, defect, glutaric aciduria type II, neonate, 663

Fluorescence quenching, peroxidase vs., plasma bilirubin binding, neonate, 349

Food protein, enterocolitis induced by, antibody response to ingested antigen, 751

Freeze-etching, biochemical analysis and, erythrocyte membranes, fetus, 1035

Galactitol, amniotic fluid, analysis, prenatal galactosemia diagnosis, 714

Galactose, diet high in, male gonad resistance to, rat, 345

Galactosemia, prenatal diagnosis, amniotic fluid galactitol analysis, 714

$\beta$-Galactosidase

developmental changes, lung, fetal and neonatal rabbit, 395

neuraminidase and, combined deficiency, 167

Gallbladder, mechanics, neonatal piglet, 1181

Gastrin, contraplacental hypogastrinemic effect, sheep, 528

Gastrointestinal tract blood flow, see Blood flow, gastrointestinal

human milk, enzyme stability, 532

Genioglossus, diaphragmatic activity and, chloral hydrate effects on, 516

Genital skin, fibroblasts, androgen receptor binding, neonate, 216

Genotype, pseudo-arylsulfatase A deficiency trait, metachromatic leukodystrophy, 1021

Gestational age

pulmonary prostaglandin biosynthetic and catabolic enzymes, ductus arteriosus, 19

small for

complement pathways, neonate, 281

glucose kinetics, 74

Glomerular filtration rate

focal segmental glomerulosclerosis and, rat, 1195

pressure gradients affecting, chronic partial ureteral obstruction, neonatal guinea pig, 1271

Glomerulosclerosis, focal segmental, rat, 1195

Glucagon, receptor, ontogeny, adenylate cyclase system, liver, neonatal guinea pig, 558

Glucocorticoid

alveolar $\beta$-adrenoreceptors, effects on, fetal rabbit, 1191

thymidine kinase activity and DNA synthesis, effects on, intestinal development, suckling rat, 145

thyroid and, interaction, lung, fetal rat, 191

Glucose

arterial and venous concentrations, uterine, pregnant guinea pig, 1172

kinetics, glucose-infused small for gestational age infant, 74

metabolism, ketone body and fatty acid effects, development, intestine, rat, 575

turnover rate, chronic catheterization, pregnancy and, rabbit, 276

Glucose-6-phosphatase, deficiency, cerebral metabolic fuel, lactate as, 335

$\beta$-Glucuronidase

deficiency, human mucopolysaccharidosis VII model, dog, 980

developmental changes, lung, fetal and neonatal rabbit, 395

$\gamma$-Glutamyl transpeptidase, deficiency, correction, amniotic fluid, cystic fibrosis, fetus, 1340

Glutaric aciduria, type II, electron transfer flavoprotein or dehydrogenase, defect, neonate, 663

Glutathione, deficiency, hereditary tyrosinemia and, 1332

Glycogenosis $\mathrm{Ib}$, neutrophil microbicidal defects, impaired hexose monophosphate shunt and, 297

Glycogen storage disease

hepatic lipase and lipoprotein lipase, activity, 881

type I, dietary treatment, slow-release carbohydrate, 879

Glycopeptide, serum, cystic fibrosis, 540

Glycoprotein neuraminidase, partial deficiency, Morquio disease type A, 302

Glycylsarcosine, uptake, malnutrition and, jejunum, rat, 504

Gonad, male, high-galactose diet, resistance to, rat, 345

Gonadotropin

human chorionic 
cyclic AMP and testosterone production, zinc ion effects, testis, rat, 232 pituitary-testicular axis responsiveness, neonate, 1085

secretion, opiate antagonism and, hypothalamic amenorrhea, 322

Granule, cytoplasmic, cord blood neutrophil metabolism, neonate, 1148

Granulocyte

circulating, $\quad N$-formyl-methionyl-leucylphenylalanine effects, vitamin $\mathrm{E}$ and, rabbit, 536

monocyte and, cord blood, 1127

Group B streptococcus, pulmonary and peripheral vascular responses to, oophorectomy and, sheep, 266

Growth

activity promoting, human milk, changes during lactation, 133

low birth weight and, longitudinal metabolic studies, neonate, 254

neonatal, fatness and serum cholesterol indices, 8-year-old, 1233

oxygen uptake and heart rate, changes, progressive exercise, 845

retardation

insulin resistance, 670

intrauterine, metabolic consequences, very low birth weight neonate, 709

maternal smoking and, rat, 127

somatomedin and cartilage activity, fetal rat, 1100

uninephrectomy effects on, chronic partial ureteral obstruction, neonatal guinea pig, 1271

Growth hormone, deficiency, fuel metabolism and, 212

Gut mucosa, barrier, food proteins and, immature and adult rats, 1252

Heart

contractile response, calcium channel blocker effects, neonatal and adult rabbit, 948

function, tachycardia, dog, 369

mitochondria, reducible cytochrome $c$ deficiency, histiocytoid cardiomyopathy, neonate, 1023

nitroprusside effects, neonatal lamb, 618

output

distribution, respiratory acidosis, fetal and neonatal lamb, 731

distribution, tolazoline effects, neonatal lamb, 896

$\mathrm{PaCO}_{2}$ variations, neonatal piglet, 1132

prostaglandin $D_{2}$ and $E_{1}$, inotropic effects neonatal rabbit, 1277

rate

abnormalities in fluctuations, sudden infant death syndrome, 921

growth-related changes, progressive exercise, 845

surgery, manganese balance studies, neonate, letter, 300

Heart ventricle

conducting system, action potential developmental changes, dog, 53

dysrhythmia, heart surgery and, dog, 1112

pacing, tachycardia induced by, cardiac function and myocardial metabolism during, dog, 369

septal defect, vasodilators and, neonatal lamb, 859

systolic time intervals, parenteral calcium for hypocalcemia and, neonate, 71

Heat, radiant vs. convective, incubators, thermoneutrality, premature neonate, 425
Height

androgen therapy and, 467

weight and, relative velocity, Benn index, 627

Hemodialysis, lipid profiles and lipase activities, chronic renal failure, 783

Hemoglobin

glycosylated, plasma protein and, diabetic mother, neonate, 767

postnatal fall, erythrocyte 2,3-diphosphoglycerate, $\mathrm{Po}_{2} 50 \%$, and available oxygen, rabbit, 154

Hemorrhage

intraventricular, cerebral blood flow and, puppy, 7

renal response, developmental aspects, fetal lamb, 40

Heparin, serum and tissue lipases, effects on, rat, 1321

Heredity, spectrin defect, hemolytic poikilocytic anemia, 1005

Herpes simplex virus, human leukocytes, antiviral antibody, and $\alpha$-interferon, ontogeny, neonatal mouse, 1164

Hexose monøphosphate, impaired shunt, neutrophil microbicidal defects due to, glycogenosis $\mathrm{Ib}, 297$

Hormone

see also specific hormone

glucocorticoid-thyroid interactions, lung, fetal rat, 191

gonadotropin-releasing, pituitary-testicular axis responsiveness, neonate, 1085

neurohypophyseal, release, hypoxia effects on, maternal and fetal sheep, 188

Howland Award

introduction, 1379

acceptance, 1383

Human milk

albumin in, iodinated compounds and thyroxine binding to, 901

collection, storage, and heating, bile saltstimulated lipase and esterase activity, 382

enzyme stability, gastrointestinal tract, 532

growth-promoting activity, changes during lactation, 133

lipases and lipids in, freeze-thawing and storage effects, 1257

Hyaline membrane disease, maternal corticosteroid therapy, fetal brain and, monkey, 440

Hybridoma antibody, group B streptococcal infection, treatment with, neonatal rat, 1093

Hydralazine, prazosin, minoxidil, and, comparison, ventricular septal defect, neonatal lamb, 859

5-Hydroxyindole acetic acid, ornithine carbamoyltransferase deficiency and, brain, mouse, 372

25-Hydroxyvitamin D, see Vitamin D

Hyperbilirubinemia, tin-protoporphyrin, prevention with, neonatal monkey, 728

Hypercapnia

cerebral blood flow and, fetal lamb, 1309

hypoxia and, retinal and choroidal blood flows, neonatal lamb, 410

Hypercarbia, see Hypercapnia

Hyperimmunoglobulin E, recurrent infection, polymorphonuclear leukocyte and monocyte inhibition, 365

Hypernatremia, rehydration, seizures after, rabbit, 340

Hyperoxia, vitamin $\mathrm{E}$ inhibition of, pulmo- nary surfactant system, neonatal rabbit, 329

Hyperreactivity, airway, cold air challenge, 469

Hypertension

cerebral blood flow, atropine effects, neonatal piglet, 1121

hypoxic pulmonary, calcium antagonist effects, piglet, 1262

Hypocalcemia, parenteral calcium, left ventricular systolic time intervals and, neonate, 71

Hypocapnia, cardiovascular system, effects on, puppy, 685

Hypocarbia, see Hypocapnia

Hypoglycemia, detection, 2-deoxyglucose test and fasting, 359, 490

Hypogonadism, hypogonadotropic, insulin resistance, 670

Hypospadias, $5 \alpha$-dihydrotestosterone binding, abnormality, neonate, 216

Hypotension, cerebral blood flow and, fetal lamb, 1309

Hypothalamus, amenorrhea, opiate antagonism and gonadotropin secretion, 322

Hypothyroidism, screening test, thyrotropin immunoassay, neonate, 1289

Hypoxanthine, oxygen and, lung injury induced by, rat, 501

Hypoxemia

aldosterone secretion, factors controlling, fetal lamb, 602

cardiac output distribution, tolazoline effects, neonatal lamb, 896

chemical sympathectomy and, cardiovascular and catecholamine responses, fetal lamb, 318

myocardial blood flows and oxygen deliveries, increased, neonatal lamb, 602

Hypoxia

cerebral blood flow and, fetal lamb, 1309

gastrointestinal blood flow and oxygen consumption, effects on, neonatal lamb, 420

hypercapnia and, retinal and choroidal blood flows, neonatal lamb, 410

lung fluid balance, neonatal lamb, 434

neurohypophyseal hormone release, effects on, maternal and fetal sheep, 188

oxygen transport and, neonatal lamb, 172

parameters, purine metabolites and lactate, neonate, 355

prostacyclin synthesis stimulated by, lung, neonatal lamb, 832

pulmonary hypertension, calcium antagonist effects, piglet, 1262

vasopressin and

amniotic fluid, fetal lamb, 552, 835

excretion, fetal vs. neonatal lamb, 227

Immune response

protein antigens, mouse, 588

respiratory syncytial virus, antibody to, 987

Immune serum globulin, group B streptococcal colonization, efficacy against, neonatal rat, 1329

Immune system, ontogeny, fetal lamb, 451

Immunoassay, enzyme, thyrotropin, hypothyroidism screening test, neonate, 1289

Immunodeficiency

combined, immunoregulation, 723

cytidine 5 -diphosphate reductase and thymidine kinase activities, phytohemagglutinin-stimulated lymphocytes, 691 
Immunoglobulin

IgG antibodies, carbohydrate and protein antigens, group B streptococci, mouse, 478

IgG and IgM, antibody responses, pneumococcal polysaccharide, neonate, 1067

Immunoreactivity, digoxin-like substance, perinatal changes, neonate, 1097

Immunoregulation, immunodeficiency, severe combined, 723

Incubator, radiant vs. convective, thermoregulation, premature neonate, 425

Indexing terms, selection, proposal, APSSPR-APA, 1205

Indomethacin

cardiopulmonary adaptations of transition, rabbit, 842

group B streptococcal sepsis, cardiovascular response to, piglet, 874

Infant, see Neonate

Infection

group B streptococcus

treatment, hybridoma antibody, neonatal rat, 1093

vascular responses, oophorectomy and sheep, 266

herpes simplex virus, protection from, ontogeny, neonatal mouse, 1164

hyperimmunoglobulin-E-associated recurrent, polymorphonuclear leukocyte and monocyte inhibition, 365

influenza B virus, acetaminophen and, Reye's syndrome, mouse, 181

$P$ seudomonas aeruginosa, agar beads and, respiratory tract, rat, 295

respiratory syncytial virus, immune response to, 987

Treponema pallidum

immunological responses, neonatal rabbit, 972

resistance to, neonatal rabbit, 965

Influenza B virus, acetaminophen and, Reye's syndrome, mouse, 181

Inspiration, external loading, effects on ventilation, premature neonates, 150

Insulin

receptor

cord blood leukocyte fluidity, 773

ontogeny, adenylate cyclase system, liver, neonatal guinea pig, 558

resistance, growth retardation, fatty liver, and hypogonadotropic hypogonadism, 670

$\alpha$-Interferon, recombinant, herpes simplex virus infection from, protection ontogeny, neonatal mouse, 1164

Intestine

bacterial adherence, postnatal age and, guinea pig, 1366

calcium transport, kinetics during maturation, rat, 235

celiac disease, cereal component toxicity, rat, 1372

development, suckling rat, 145

glucose metabolism, ketone body and fatty acid effects, development, rat, 575

microvillus membrane, differentiation, cholera toxin binding, newborn and adult rabbits, 984

pro-sucrase-isomaltase, high molecular weight, fetus, letter, 391

secretion, bilirubin-induced, reversal by agar, hamster, 79

small

biopsies, diamine oxidase and disaccharidase activities, 647

upper, lipolysis mechanism, rat, 402
Iodine, binding to albumin, human milk, 901 Iron

deficiency

diagnostic tests, receiver-operator analysis, 916

neutrophil bactericidal dysfunction, $\mathrm{ox}$ idant radical-sensitive microorganisms, rat, 684, 789

neutrophils and, rat, 549

oxidative metabolism, skeletal muscle, rat, 499

maternal supplements, fetal lung catalase activity, rat, 871

skeletal muscle, ferritin in, vitamin $\mathrm{E}$ deficiency, rabbit, 1012

Isoelectric focusing, serum and plasma, cystic fibrosis protein detection, 130

Isoproterenol, pulmonary function, cystic fibrosis, rat, 1029

Isovaleryl glucuronide, excretion pattern, isovaleric acidemia and, 508

Jaundice

bilirubin concentrations, neonate, letter, 682

phototherapy, light source comparison, neonate, 667

Jejunum

L-leucine and glycylsarcosine uptake, malnutrition, rat, 504

methylated casein, anticholeraic effect, rat, 1075

mucous coat and surface membrane, cow's milk protein, interactions with, immature and adult rat, 1252

$\alpha$-Ketoacid, branched chain amino acids and, maple syrup urine disease, neonate, 851

Ketogenesis, fatty acid, hepatic, neonatal guinea pig, letter, 299

Ketone

arterial and venous concentrations, uterine, pregnant guinea pig, 1172

fatty acid and, intestinal glucose metabolism, development, rat, 575

6-Ketoprostaglandin $F_{1 \alpha}$, prenatal dexamethasone, effects, lung, fetal rat, 908

Kidney

$\beta$-amino acid transport, development, rat 611

anion transport, Mendelian mutation and human and mouse, 25

blood flow, see Blood flow, renal

cortical tubules, sugar transport, development, dog, 719

failure, chronic, lipid profiles and lipase activities, 783

hepatorenal tyrosinemia, glutathione deficiency, 1332

osteodystrophy, continuous ambulatory peritoneal dialysis, 742

plasma flow, focal segmental glomerulosclerosis and, rat, 1195

\section{Lactate}

arterial and venous concentrations, uterine, pregnant guinea pig, 1172

cerebral metabolic fuel, glucose-6-phosphatase deficiency and, 335

purine metabolites and, hypoxia parameters, neonate, 355

Lactation, growth-promoting activity, changes in, human milk, 133

Learning disability, early malnutrition and,

Letters to the Editor, 299, 390, 496, 570, 682

L-Leucine, uptake, malnutrition and, je- junum, rat, 504

Leukocyte

enzyme source, acetyl-CoA: $\alpha$-glucosamine $N$-acetyltransferase assay, Sanfilippo syndrome, 543

human, herpes simplex virus infection from, protection ontogeny, neonatal mouse, 1164

mononuclear, cord blood, membrane fluid properties, increased insulin receptors and, 773

polymorphonuclear, chemotactic inhibition, hyperimmunoglobulin-E-associated infection, 365

very long chain fatty acids, impaired oxidation, adrenoleukodystrophy, 286

Leukodystrophy, metachromatic, pseudoarylsulfatase A deficiency without, genotypes, 1021

Lipase

activities, lipid profiles and, chronic renal failure, 783

bile salt-stimulated activity, collected, stored, and heated human milk, 382

hepatic, glycogen storage disease, 881

human milk, freeze-thawing and storage effects, 1257

lingual

fat digestion, stomach and upper small intestine, rat, 402

stability, gastric environment, rat, 248

plasma, postheparin, plasma lipoproteins and, neonate, 642

plasma and hepatic, postheparin, premature neonate, 1104

serum and tissue, heparin effects, rat, 1321

Lipid

emulsion, intravenous, essential fatty acid deficiency, neonatal pig, 1350

human milk, freeze-thawing and storage effects, 1257

Menkes' disease, neonate, 864

profiles, lipase activities and, chronic renal failure, 783

Lipolysis, mechanism, stomach and upper small intestine, rat, 402

Lipoprotein

Menkes' disease, neonate, 864

plasma

postheparin, premature neonate, 1104

postheparin plasma lipase activities and, neonate, 642

Lipoprotein lipase, hepatic lipase and, glycogen storage disease, 881

Liver

adenylate cyclase system, insulin and glucagon receptors, ontogeny, neonatal guinea pig, 558

failure, sodium valproate and, letter, 390

fatty, insulin resistance, 670

fatty acid oxidation and ketogenesis, neonatal guinea pig, letter, 299

hepatorenal tyrosinemia, glutathione deficiency, 1332

lipase

glycogen storage disease, 881

premature neonate, 1104

$l$-Norepinephrine, pulmonary and femoral arteries, stimulation by, neonatal lamb, 244

Lung

antioxidant enzymes

birth into oxygen-rich environment, rabbit, 240

maturation, fetal and neonatal rat, 584, 871

chronic ventilation, respiratory distress syndrome, type I/III collagen ratios, 
neonate, 1176

developing, muscarinic cholinergic receptors, rat, 1136

dipalmitoylphosphatidylcholine content and phosphatidylcholine species composition, betamethasone us. Lcarnitine effects, fetal rat, 1246

distensibility, airway function and asthma, 1154

enzymes, developmental changes, fetal and neonatal rabbit, 395

fluid balance, hypoxia, neonatal lamb, 434

glucocorticoid-thyroid hormone interactions, fetal rat, 191

group B streptococcus infusion, vascular responses, oophorectomy and, sheep, 266

hypertension, hypoxic, calcium antagonist effects, piglet, 1262

injury, hypoxanthine and oxygen induced, rat, 501

6-ketoprostaglandin $\mathrm{F}_{1 \alpha}$ levels, prenatal dexamethasone effects, fetal rat, 908

liquid absorption and alveolar epithelial solute permeability, surfactant deficiency, fetal lamb, 566

liquid ventilation, meconium-stained lamb, 47

maturation, intra-amniotic triiodothyronine, effects on, fetal lamb, 932

prostacyclin synthesis, hypoxia-stimulated, neonatal lamb, 832

prostaglandin biosynthetic activities, fetal lamb, 12

prostaglandin biosynthetic and catabolic - enzymes, ductus arteriosus patency and closure, fetal lamb, 19

reserpine and isoproterenol models, cystic fibrosis, rat, 1029

retinol and retinyl palmitate, perinatal rat, 1297

secretions, $\alpha_{1}$-proteinase inhibitor and $\alpha_{2}$ macroglobulin in, intubated neonate, 35

surfactant system, hyperoxia effects, vitamin $\mathrm{E}$ inhibition of, neonatal rabbit, 329

vascular prostacyclin synthesis, onset of breathing at birth, lamb and goat, 938

vascular resistance, meclofenamate effects on, piglet, 579

volume calculation, nitrogen washout method, neonate, 1160

Lymphocyte

alloantigen, response to, neonate, 414

cystic fibrosis, methylprednisolone resist. ance of, 488

peripheral blood, $\mathrm{Cl}^{-}$permeabilities, cystic fibrosis, 1336

phytohemagglutinin-stimulated, cytidine 5 '-diphosphate reductase and thymidine kinase activities, immunodeficiency, 691

Lysosome, disease, diagnosis, first trimester, trophoblast biopsy, 1032

$\alpha_{2}$-Macroglobulin

cystic fibrosis, monoclonal antibodies and, 999

$\alpha_{1}$-proteinase inhibitor and, concentrations, serum and lung secretions, intubated neonate, 35

Macrophage, skeletal muscle, iron sequestration in, vitamin E deficiency, rabbit, 1012

Malnutrition

early behavioral development and, soft neurologic signs, 826

learning disabilities and, 309

endocrine adaptation, letter, 300

intrauterine, growth retardation, metabolic consequences, very low birth weight neonate, 709

L-leucine and glycylsarcosine uptake, jejunum, rat, 504

protein-calorie, plasma and red blood cell fatty acid composition, 162

protein-energy, theophylline effects, brain, neonatal rat, 546

Manganese, balance studies, heart surgery and, neonate, letter, 300

Maternity

age, Down's syndrome and, mathematical function, 375

diabetes, glycosylated hemoglobin and plasma protein, neonate, 767

fetus, neurohypophyseal hormone release, hypoxia effects on, sheep, 188

hyaline membrane disease, corticosteroid therapy, fetal brain and, monkey, 440

iron supplements, fetal lung catalase activity, rat, 871

smoking

fetal growth retardation and, rat, 127

xenobiotic metabolism, placenta, 1071

suppressor T-cell activity, human mother and neonate, 123

water transfer to fetus, cortisol effects, lamb, 631

Maturation, intestinal calcium transport, kinetics during, rat, 235

Meclofenamate, pulmonary vascular resistance, effects on, piglet, 579

Meconium, stain, liquid ventilation and pulmonary function, lamb, 47

Menkes' disease, lipids, lipoproteins, and apoliproteins, neonate, 864

Metabolic rate, low birth weight and, longitudinal studies, neonate, 254

Metallothionein, inducers, trace metal metabolism in cultured skin fibroblasts mouse, 1282

Methylprednisolone

cystic fibrosis lymphocytes, resistance to, 488

group B streptococcal toxin, response to, sheep, 1141

Microvillus membrane, differentiation, cholera toxin binding, intestine, newborn and adult rabbits, 984

Milk

cow's, mucous coat and surface membrane, interactions with, jejunum, immature and adult rat, 1252

human, see Human milk

monkey, composition, 911

Mineral

blood, circadian rhythms, adolescence, 456

composition, monkey milk, 911

tissue, sudden infant death syndrome and letter, 570

Minoxidil, prazosin, hydralazine, and, comparison, ventricular septal defect, neonatal lamb, 859

Mitochondria, myopathy, respiratory complex III component deficiency, 991

Mitochondrial membrane, abnormalities, calcium metabolism and, cystic fibrosis, 594

Monocyte, chemotactic inhibition, hyperimmunoglobulin-E-associated infection, 365

monocyte, granulocyte and, cord blood, 1127
Morquio disease type A, glycoprotein neuraminidase, partial deficiency, 302

Mucopolysaccharidosis VII, human, model,

Muscle $\beta$-glucuronidase deficiency, dog, 980

skeletal

iron sequestration in, vitamin $\mathrm{E}$ deficiency, rabbit, 1012

oxidative metabolism in, iron deficiency effects, rat, 499

upper airway, regulation, progressive asphyxia, rabbit, 819

Mutation, Mendelian, renal sulfate and phosphate transport, human and mouse, 25

Myocarditis, diphtheric, carnitine protective effect, 815

Myocardium

blood flow, see Blood flow, myocardial

excitation-contraction coupling, alloxandiabetic, fetal rabbit, 1344

hypoxemia, increased blood flow and oxygen deliveries, neonatal lamb, 602

metabolism, tachycardia, dog, 369

Myopathy, mitochondrial, respiratory complex III component deficiency, 991

Needle, intravenous, bacterial adherence to, phlebitis and, rabbit, 1361

Neonate

adenylate cyclase system, insulin and glucagon receptors, ontogeny, liver, guinea pig, 558

adult and

monovalent cation transport, erythrocyte, 778

platelet thromboxane production differences, 823

auditory brainstem response, 780

bilirubin binding, plasma, fluorescence quenching us. peroxidase, 349

cardiopulmonary function, measurement, rebreathing methodology, piglet, 1164

carnitine, tissue reserves, 675

cerebral blood flow

electrical impedance vs. strain gauge plethysmography, 290

pancuronium bromide and, lamb, 1305

chemotactic peptide binding, intact neutrophils, 63

chronically ventilated, respiratory distress syndrome, type I/III collagen ratios, 1176

cord blood neutrophils, oxidative metabolism, cytoplasmic granules and, 1148

critically ill

carbonic acid dissociation constant, 1287

physiologic stability index, 445

epidermal growth factor, urine, mouse, 1080

Escherichia coli $\mathrm{K} 1$ sepsis, antibiotic therapy, rabbit, 314

essential fatty acid deficiency, intravenous lipid emulsions, pig, 1350

eupnea, maturation, monkey, 943

fasting, splanchnic uptake and energy substrate release, baboon, 1316

fatty acid oxidation and ketogenesis, hepatic, guinea pig, letter, 299

feeding and growth, fatness and serum cholesterol indices, 8-year-old, 1233

fetus and

hypoxia, vasopressin excretion, lamb, 227

lung enzyme activities, developmental 
changes, rabbit, 395

pulmonary antioxidant enzyme maturation, rat, 584,871

respiratory acidosis, cardiac output distribution, lamb, 731

thromboxane $\mathrm{B}_{2}$ production, respiratory distress syndrome and birth asphyxia, 756

gallbladder mechanics, piglet, 1181

gastrointestinal blood flow and oxygen consumption, anemia and hypoxia effects on, lamb, 420

glucose-infused small for gestational age, glucose kinetics, 74

glutaric aciduria type II, electron transfer flavoprotein or dehydrogenase, defect, 663

group B streptococcus

colonization, immune serum globulin efficacy against, rat, 1329

infection, treatment, hybridoma antibody, rat, 1093

heart

prostaglandin $D_{2}$ and $E_{1}$, inotropic effects, rabbit, 1277

surgery, manganese balance studies, letter, 300

herpes simplex virus, protection from, ontogeny, mouse, 1164

histiocytoid cardiomyopathy, reducible cytochrome $c$ deficiency, 1023

25-hydroxyvitamin D and total calcium, concentrations, Saudi Arabia, 739

hyperbilirubinemia, prevention, tin-protoporphyrin, monkey, 728

hypocalcemia, parenteral calcium and, left ventricular systolic time intervals, 71

hypothyroidism, screening test, thyrotropin immunoassay, 1289

hypoxemia

cardiac output distribution, tolazoline effects, 896

increased myocardial blood flows and oxygen deliveries, lamb, 602

hypoxia

lung fluid balance, lamb, 434

oxygen transport, lamb, 172

parameters, purine metabolites and lactate, neonate, 355

hypoxia and hypercapnia, retinal and choroidal blood flows, lamb, 410

idiopathic respiratory distress syndrome, antithrombin III levels and prognosis, 273

intubated, serum and lung secretions, $\alpha_{1}$ proteinase inhibitor and $\alpha_{2}$-macroglobulin, 35

jaundice

bilirubin photo-oxidation products, phototherapy and, 696

phototherapy for, light source comparison, 667

serum bilirubin concentrations, letter, 682

l-norepinephrine stimulation, pulmonary and femoral arteries, lamb, 244

low birth weight, longitudinal metabolic studies, 254

lung volume, calculation, nitrogen washout method, 1160

lymphocytes, response to alloantigen, 414 meconium-stained, liquid ventilation and pulmonary function, lamb, 47

mother and, suppressor T-cell activity, 123

nitrogen metabolism, infant nutrition model, pig, 658
$\mathrm{PaCO}_{2}$, variations, brain blood flow and cardiac output, piglet, 1132

pneumococcal immunization, 1067

postheparin plasma lipases, plasma lipoproteins and, 642

premature, see Prematurity

prenatal thyroid function abnormalities, idiopathic respiratory distress syndrome and, 926

prostacyclin synthesis, hypoxia-stimulated, lungs, lamb, 832

protein-energy malnourished, theophylline and cellular response, brain, rat, 546

pulmonary surfactant system, hyperoxia effects, vitamin $\mathrm{E}$ inhibition of, rabbit, 329

reserpine, chronic treatment, effects on pancreas, rat, 1300

small for gestational age and preterm, complement pathways, 281

syphilis

immunological responses, rabbit, 972

resistance to, rabbit, 965

term and preterm

erythrocyte aggregation, 1356

sleep states, human, 58

$\alpha$-thalassemia-2, deletion and nondeletion, 158

tryptophan, cholestatic effect, 904

ureteral obstruction

chronic partial, pressure gradients and glomerular filtration rate, guinea pig, 1271

chronic partial, uninephrectomy effects, guinea pig, 1266

ventilation depression, adenosine analogues, rabbit, 387

very low birth weight, intrauterine growth retardation, metabolic consequences, 709

Nephritis, phagocyte adherence, reduction by, 637

Neuraminidase, $\beta$-galactosidase and, combined deficiency, 167

Neutrophil

activation and metabolism, iron deficiency and, rat, 549

bactericidal dysfunction, oxidant radicalsensitive microorganisms, iron deficiency, rat, 684, 789

cord blood

monoclonal antibody 2-2-B and, Escherichia coli $\mathrm{K} 1$, effects on, mouse, 770

oxidative metabolism, cytoplasmic granules and, neonate, 1148

group B streptococcus, bactericidal capacity, neonate, 634

intact, chemotactic peptide binding, neonate, 63

microbicidal defects, impaired hexose monophosphate shunt, and glycogenosis Ib, 297

$N$-Formyl-methionyl-leucyl-phenylalanine, vitamin $\mathbf{E}$ and, circulating granulocytes, rabbit, 536

Nieman-Pick disease, types A, B, and C, fibroblasts, sphingomyelinase activity in, 1088

Nifedipine, heart contractile response, effects on, neonatal and adult rabbit, 948

Nitrogen, metabolism, infant nutrition model, neonatal pig, 658

Nitrogen washout test, lung volume calculation, neonate, 1160

Nitroprusside, circulatory effects, neonatal lamb, 618
Nutrition

casein and whey proportions, nitrogen metabolism and digesta composition, neonatal pig, 658

human milk, collection, storage, and heating, lipase and esterase activity, 382

total parenteral cysteine-supplemented, puppy, 747

fuel mixes, body composition and, neonatal miniature pig, 261

Oligosaccharide, ciliary dyskinetic activity and, cystic fibrosis, 540

Ontogeny

herpes simplex virus infection, protection from, neonatal mouse, 1164

immune system, fetal lamb, 451

insulin and glucagon receptors, adenylate cyclase system, liver, neonatal guinea pig, 558

Oophorectomy, pulmonary and peripheral vascular responses, group B streptococcus and, sheep, 266

Opiate, antagonism, gonadotropin secretion and, hypothalamic amenorrhea, 322

Organic acid, metabolism, disorder, L-carnitine secondary insufficiency, 1325

Ornithine carbamoyltransferase, deficiency, tryptophan and 5-hydroxyindole acetic acid increase, brain, mouse, 372

Oscillation, modified forced, respiratory resistance measurement, 139

Osteodystrophy, renal, continuous ambulatory peritoneal dialysis, 742

Osteogenesis tarda, increased bone turnover with decreased bone formation, letter, 497

Oxidative metabolism

iron deficiency effects, skeletal muscle, rat, 499

neutrophil cord blood, cytoplasmic granules and, neonate, 1148

Oxygen

arterial and venous concentrations, uterine, pregnant guinea pig, 1172

consumption

anemia and hypoxia effects on, neonatal lamb, 420

nitroprusside effects, neonatal lamb, 618

premature neonate, 928

environment rich in, birth into, antioxidant enzymes, lung, rabbit, 240

hypoxanthine and, lung injury induced by, rat, 501

increased deliveries, myocardium, hypoxemia and, neonatal lamb, 602

transport, hypoxic neonatal lamb, 172

uptake, growth-related changes, progressive exercise, 845

Oxygenation, arterial, cerebral blood flow and, fetal lamb, letter, 572

Oxygen pressure, $\mathrm{Po}_{2} 50 \%$, erythrocyte 2,3 diphosphoglycerate and, postnatal fall in hemoglobin, rabbit, 154

Paediatric Pathology Society, European Society for Paediatric Research, and Working Group on Mineral Metabolism, annual meeting abstracts, 795

Pancreas

exocrine function, carbohydrate tolerance and, cystic fibrosis, 1107

insufficiency, calcium-ATPase activity, erythrocyte membranes, cystic fibrosis, 890 
reserpine, chronic treatment, effects on, neonatal rat, 1300

Pancuronium bromide, cerebral blood flow, effects on, neonatal lamb, 1305

Peptide, chemotactic, binding, intact neutrophils, neonate, 63

Perinate

immunoreactive substance, digoxin-like, changes, 1097

retinol and retinyl palmitate, lung, rat, 1297

Perinatology, advances, Clinical Research Centers, 197

Permeability

alveolar epithelial solute, surfactant deficiency, lung, fetal lamb, 566

$\mathrm{Cl}^{-}$, erythrocytes and peripheral blood lymphocytes, cystic fibrosis, 1336

Peroxidase, fluorescence quenching us., plasma bilirubin binding, neonate, 349

Pertussis, vaccine, insulin-dependent diabetes and, mouse, 221

$\mathrm{pH}$, bilirubin photo-derivative water solubility, relevance to phototherapy, 378

Phagocyte, adherence, nephritic sera, 637

Phlebitis, intravenous catheters and needles, bacterial adherence to, rabbit, 1361

Phosphatidylchloline, species composition, betamethasone vs. L-carnitine effects, lung, fetal rat, 1246

Phospholipase $A_{1}$ and $A_{2}$, developmental changes, lung, fetal and neonatal rabbit, 395

Phospholipid, erythrocyte membrane, abnormal fatty acid turnover, cystic fibrosis, 704

Photo-derivative, bilirubin, water solubility of, $\mathrm{pH}$ dependence, phototherapy and, 378

Phototherapy

bilirubin photo-derivatives, water solubility of, $\mathrm{pH}$ dependence, 378

DNA damage, enhancement by bilirubin, human, 3

jaundice, bilirubin photo-oxidation products, urine, neonate, 696

light source comparison, jaundice, neonate, 667

Phytohemagglutinin, lymphocytes stimulated by, cytidine $5^{\prime}$-diphosphate reductase and thymidine kinase activities, immunodeficiency, 691

Pituitary-testicular axis, gonadotropin-releasing hormone and chorionic gonadotropin, responsiveness to, neonate, 1085

Placenta

blood flow, see Blood flow, placental

gastrin infusion and, sheep, 528

somatostatin binding, plasma cell membrane, 953

xenobiotic metabolism, maternal smoking and, 1071

Plasma

bilirubin binding, fluorescence quenching vs. peroxidase, neonate, 349

composition, protein-calorie malnutrition, 162

flow, renal, focal segmental glomerulosclerosis and, rat, 1195

25-hydroxyvitamin D and total calcium concentrations, Saudi Arabian mothers and neonates, 739

isoelectric focusing, cystic fibrosis protein detection, 130

lipase, postheparin, premature neonate, 1104 lipoprotein, postheparin plasma lipase activities and, neonate, 642

membrane, placenta, somatostatin binding, 953

protein, glycosylated hemoglobin and, diabetic mother, neonate, 767

somatomedin activity, reduced, growth retardation, fetal rat, 1100

vitamin $D$, exchange transfusions with citrated blood, effects on, neonate, 429

vitamin D metabolite concentration, premature neonate, 269

Platelet

Down's syndrome, decreased calcium content and ${ }^{45} \mathrm{Ca}^{2+}$ uptake, 914

thromboxane production, arachidonic acid and epinephrine stimulation, neonate and adult, 823

Plethysmography, strain gauge, electrical impedance $v s$, cerebral blood flow study, neonate, 290

Poikilocyte, anemia, hemolytic, spectrin defect, 1005

Polysaccharide, pneumococcal, IgG and IgM, antibody responses, neonate, 1067

Prazosin, minoxidil, hydralazine, and, comparison, ventricular septal defect, neonatal lamb, 859

Pregnancy

chronic catheterization, glucose turnover rates and, rabbit, 276

diabetes in, placental blood flow, rat, 735

maternal metabolism, rabbit, 854

substrate-oxygen blood levels, uterine, guinea pig, 1172

Prematurity

body water measurements, neonate, 524

complement pathways, alternative, 281

6-keto-prostaglandin $\mathrm{F}_{1 \alpha}$ excretion, respiratory distress and, 520

postheparin plasma lipases, neonate, 1104

respiratory metabolism, oxygen consumption, neonate, 928

term and

erythrocyte aggregation, neonate and adult, 1356

sleep states, 58

thermoneutrality, radiant vs. convective heat incubation, 425

ventilation, external inspiratory loading, 150

vitamin D metabolites, plasma concentrations, 269

Presidential Address, American Pediatric Society 1984, 1202

Preterm, see Prematurity

Prolidase, deficiency, prolidase defect and, congenital expression, 259

Prostacyclin

group B streptococcal sepsis, cardiovascular relationship to, piglet, 874

synthesis

hypoxia-stimulated, lungs, neonatal lamb, 832

pulmonary vascular, onset of breathing at birth, lamb and goat, 938

Prostaglandin

biosynthetic activities, ductus arteriosus, blood vessels, and lung tissue, fetal lamb, 12

$D_{2}$ and $E_{1}$, inotropic effects, heart, neonatal rabbit, 1277

enzymes, biosynthetic and catabolic, ductus arteriosus patency and closure, fetal lamb, 19

urinary excretion rate, respiratory distress recovery, premature neonate,
520

Pro-sucrase-isomaltase, high molecular weight, intestine, human fetus, letter, 391

Protein

antigen

IgG antibodies to, group B streptococci, mouse, 478

immune response, mouse, 588

composition, monkey milk, 911

excretion, focal segmental glomerulosclerosis and, rat, 1195

food, gut mucosal barrier and, immature and adult rats, 1252

plasma, glycosylated hemoglobin and, diabetic mother, neonate, 767

$\alpha_{1}$-Proteinase inhibitor, $\alpha_{2}$-macroglobulin and, concentrations, serum and lung secretions, intubated neonate, 35

Pseudo-arylsulfatase A

deficiency

diagnosis, electophoresis, 1042

genotypes, 1021

Pseudomonas aeruginosa

mucoid exopolysaccharide, antibody to cystic fibrosis serum, 431

pathogenicity, agar bead role in, respiratory tract, rat, 295

Purine, metabolite, lactate and, hypoxia parameters, neonate, 355

Pyruvate, metabolism, dichloroacetate effects, brain, rat, 936

Receptor

adenosine, theophylline stimulation of respiration via, neonatal rabbit, 387 insulin

cord blood leukocyte fluidity, 773

glucagon, ontogeny, adenylate cyclase system, liver, neonatal guinea pig, 558

muscarinic cholinergic, developing lung, rat, 1136

Red blood cell, see Erythrocyte

Rehydration, hypernatremia, seizures after, rabbit, 340

Reserpine

chronic treatment, pancreas, effects on neonatal rat, 1300

pulmonary function, cystic fibrosis, rat, 1029

Respiration

eupneic, maturation, neonatal monkey, 943

metabolism, premature neonate, 928

onset at birth, pulmonary vascular prostacyclin synthesis, lamb and goat, 938

resistance, measurement, modified forced oscillation method, 139

theophylline stimulation of, adenosine receptors, neonatal rabbit, 387

Respiratory distress syndrome

birth asphyxia and, thromboxane $B_{2}$ production, neonate and fetus, 756

chronic ventilation, type I/III collagen ratios and, neonate, 1176

idiopathic

antithrombin III levels and prognosis, neonate, 273

prenatal thyroid function abnormalities, neonate, 926

patent ductus arteriosus and, recovery, prostaglandin excretion rates, premature neonate, 520

Respiratory syncytial virus, immune response to, 987

Respiratory system

acidosis, cardiac output distribution, fetal 
and neonatal lamb, 731

complex III component deficiency, lactic acid and mitochondrial myopathy, 991

heart rate and, abnormalities in fluctuations, sudden infant death syndrome, 921

Pseudomonas aeruginosa, pathogenicity, agar beads and, rat, 295

Retina, blood flow, see Blood flow, retinal

Retinol, retinyl palmitate and, lung, perinatal rat, 1297

Retinyl palmitate, retinol and, lung, perinatal rat, 1297

Reye's syndrome, influenza B virus and acetaminophen, mouse, 181

Sanfilippo syndrome, type C, acetyl-CoA: $\alpha$ glucosamine $N$-acetyltransferase assay, 543

Saudi Arabia, mothers and neonates, 25hydroxyvitamin $\mathrm{D}$ and total calcium concentrations, 739

Seizure, rehydration and, hypernatremia treatment, rabbit, 340

Sepsis

bacterial, granulocytic stem cell development, rat, 599

Escherichia coli $\mathrm{K} 1$, antibiotic therapy, neonatal rabbit, 314

group B streptococcus, cardiovascular changes, piglet, 874

Serum

soelectric focusing, cystic fibrosis protein detection, 130

$\alpha_{1}$-proteinase inhibitor and $\alpha_{2}$-macroglobulin in, intubated neonate, 35

Shigella flexneri, adherence, postnatal age and, intestine, guinea pig, 1366

Shunt, hexose monophosphate, impaired, neutrophil microbicidal defects due to, glycogenosis Ib, 297

Skin, fibroblast, trace metal metabolism, mouse, 1282

Sleep

organization, sudden infant death syndrome, 654

states, term and preterm infants, 58

Smoking

maternal

fetal growth retardation and, rat, 127

xenobiotic metabolism, placenta, 1071

Sodium alginate, antibody to, cystic fibrosis serum, 431

Sodium valproate, liver failure and, letter, 390

Solubility, bilirubin photo-derivatives, $\mathrm{pH}$ dependence of, phototherapy and, 378

Somatomedin, reduced plasma activity, growth retardation, fetal rat, 1100

Somatostatin, binding, plasma cell membranes, placenta, 953

Spectrin, defect, hemolytic poikilocytic anemia, 1005

Sphingomyelinase, activity, Niemann-Pick type A, B, and C fibroblasts, 1088

Spinal fluid, monoclonal antibody 2-2-B and, Escherichia coli $\mathrm{K} 1$, effects on, mouse, 770

Splanchnic uptake, energy substrate release, fasting, neonatal baboon, 1316

Stability index, physiologic, critically ill neonates and children, 445

Stem cell, granulocytic, pre- and postnatal development, rat, 599

Stomach

digesta, nitrogen metabolism and, casein and whey proportions, neonatal pig,
658

fat digestion, lingual lipase stability, rat, 248

upper small intestine and, lipolysis mechanism, rat, 402

Streptococcus

group B

bacteremia, tolazoline in, neonatal piglet, 960

bactericidal capacity, neutrophils, neonate, 634

IgG antibodies to carbohydrate and protein antigens, mouse, 478

methylprednisolone effects on, sheep, 1141

sepsis, cardiovascular changes, piglet, 874

treatment, hybridoma antibody, neonatal rat, 1093

type III, immune serum globulin efficacy against, neonatal rat, 1329

Streptozotocin, diabetes induced by, pertussis virus effects, mouse, 221

Substrate, energy, release, splanchnic uptake and, fasting, neonatal baboon, 1316

Sudden infant death syndrome

heart rate and respiratory activity, abnormalities in fluctuations, 921

nocturnal sleep organization, 654

tissue mineral levels, letter, 570

Sugar, transport, renal cortical tubules, development, dog, 719

Sulfate, costal cartilage, reduced activity, growth retardation, fetal rat, 1100

Sulfhydryl oxidase, human milk, stability, gastrointestinal tract, 532

Sulfur, amino acids, altered intake, brush border membrane response, rat, 611

Surfactant

deficiency, liquid absorption and alveolar epithelial solute permeability, fetal lamb, 566

hyperoxia effects, vitamin $\mathrm{E}$ inhibition of, neonatal rabbit, 329

Surgery, heart, ventricular dysrhythmias after, dog, 1112

Sweat duct, ion-selective and transport properties, cystic fibrosis sweat factor, apparent absence, 1292

Sweat gland, anion exchange mechanism, abnormal, cystic fibrosis, letter, 496

Sympathectomy, chemical, hypoxemia, cardiovascular and catecholamine responses, fetal lamb, 318

Syncytia, respiratory syncytial virus, antibody to, 987

Syphilis

Treponema pallidum inoculation immunological responses, neonatal rabbit, 972

resistance to, neonatal rabbit, 965

Tachycardia, atrial and ventricular pacing causing, cardiac function and myocardial metabolism during, dog, 369

T-cell, suppressor, neonate and mother, 123

Testes

cyclic AMP and testosterone production, human chorionic gonadotropin-stimulated, effects on, rat, 232

pituitary-testicular axis, gonadotropin-releasing hormone and chorionic gonadotropin, responsiveness to, neonate, 1085

Testosterone

biosynthetic effects, evaluation, 759

human chorionic gonadotropin-stimulated production, zinc ion effects, tes- tis, rat, 232

$\alpha$-Thalassemia-2, deletion and nondeletion, neonate, 158

Theophylline

cellular response, malnourished brain, neonatal rat, 546

respiration stimulation, adenosine receptors, neonatal rabbit, 387

Thermoregulation, incubator, radiant us convective, premature neonate, 425

Thromboxane, production, platelets, arachidonic acid and epinephrine stimulation, neonate and adult, 823

Thromboxane $A_{2}$, group $B$ streptococcal sepsis, cardiovascular relationship to, piglet, 874

Thromboxane $\mathrm{B}_{2}$, production, respiratory distress syndrome and birth asphyxia, effects, fetus and neonate, 756

Thymidine kinase

activity, weaning, diet composition, and glucocorticoid effects on, intestinal development, suckling rat, 145

phytohemagglutinin-stimulated lymphocytes, immunodeficiency and, 691

Thyroid, glucocorticoid and, interaction, lung, fetal rat, 191

Thyroid gland, function abnormalities, prenatal, idiopathic thyroid function abnormalities and, neonate, 926

Thyrotropin, enzyme immunoassay, hypothyroidism screening test, neonate, 1289

Thyroxine

binding to albumin, human milk, 901

maturative effect, epidermal growth factor, urine, neonatal mouse, 1080

Tin-protoporphyrin, hyperbilirubinemia, prevention with, neonatal monkey, 728

Tolazoline

cardiac output distribution, effects on, hypoxemia, neonatal lamb, 896

group B streptococcal bacteremia, hemodynamics, neonatal piglet, 960

Trace metal, metabolism, skin fibroblasts, mouse, 1282

Transplantation, kidney, lipid profiles and lipase activities, chronic renal failure, 783

Treponema pallidum

immunological responses, neonatal rabbit, 972

resistance to, neonatal rabbit, 965

Triglyceride, metabolism, Wolman's disease and cholesterol ester storage disease, 1242

Triiodothyronine, intra-amniotic, pulmonary maturation, effects on, fetal lamb, 932

Trophoblast, biopsy, lysosomal disease diagnosis, first trimester, 1032

Trypsin, immunoreactive, amniotic fluid, cystic fibrosis, fetus, 957

Tryptophan

cholestatic effect, neonatal rat, 904

ornithine carbamoyltransferase ciency and, brain, mouse, 372

Tyrosine aminotransferase, low activity, persistent tyrosinemia and, 675

Tyrosinemia

hereditary hepatorenal, glutathione deficiency, 1332

persistent, low tyrosine aminotransferase activity and, 675

type I, enzyme defect, 463

Umbilical vein, endothelium, maternal 
smoking effects, 1071

Uninephrectomy, chronic partial ureteral obstruction, growth and hemodynamics, neonatal guinea pig, 1271

Ureter, obstruction, chronic partial, pressure gradients and glomerular filtration rate, neonatal guinea pig, 1266

Urine

bilirubin photooxidation products in, phototherapy and, jaundice, neonate, 696

L-carnitine and acylcarnitine excretion, organic acid metabolism disorders, 1325

dicarboxylic acid, identification, propionic and methylmalonic acidemia, 1185

epidermal growth factor, thyroxine effects, neonatal mouse, 1080

isovaleryl glucuronide excretion, isovaleric acidemia and, 508

prostaglandin excretion rate, respiratory distress recovery, premature neonate, 520

Uterus, substrate-oxygen blood levels, pregnant guinea pig, 1172

Vaccine, pertussis, insulin-dependent diabetes and, mouse, 221

Vasodilator, prazosin, minoxidil, and hydralazine, ventricular septal defect, neonatal lamb, 859
Vasopressin

amniotic fluid, concentrations in, hypoxia index, fetal lamb, 552, 835

arginine, blood pressure control, rat, 701

excretion, hypoxia and, fetal and neonatal lamb, 227

Ventilation

chronic, respiratory distress syndrome, type I/III collagen ratios, neonate, 1176

depression, adenosine analogues, neonatal rabbit, 387

external inspiratory loading, effects on, premature neonate, 150

liquid, pulmonary function and, meconium-stained lamb, 47

Ventricle, heart, see Heart ventricle

Verapamil, heart contractile response, effects on, neonatal and adult rabbit, 948

Vitamin A, retinyl palmitate and, lung, perinatal rat, 1297

Vitamin D

25-hydroxyvitamin D, total calcium and, concentrations, Saudi mothers and neonates, 739

metabolite

levels, 886

plasma concentrations, exchange transfusions with citrated blood, neonate, 429

plasma concentrations, premature neonate, 269
Vitamin E

deficiency, iron sequestration in skeletal muscle, rabbit, 1012

hyperoxia inhibition, pulmonary surfactant system, neonatal rabbit, 329

$N$-formyl-methionyl - leucyl - phenylalanine, effects on, circulating granulocytes, rabbit, 536

Water transfer, fetal/maternal, cortisol effects, lamb, 631

Weaning, thymidine kinase activity and DNA synthesis, effects on, intestinal development, suckling rat, 145

Weight, height and, relative velocity, Benn index, 627

Whey, casein and, different proportions, nitrogen metabolism, neonatal pig, 658

White blood cell, see Leukocyte

Wolman's disease, cholesterol ester and triglyceride metabolism, fibroblasts, 1242

Working Group on Mineral Metabolism European Society for Paediatric Research, and Paediatric Pathology Society, annual meeting abstracts, 795

Zinc, cyclic AMP and testosterone production, human chorionic gonadotropinstimulated, effects on, testis, rat, 232

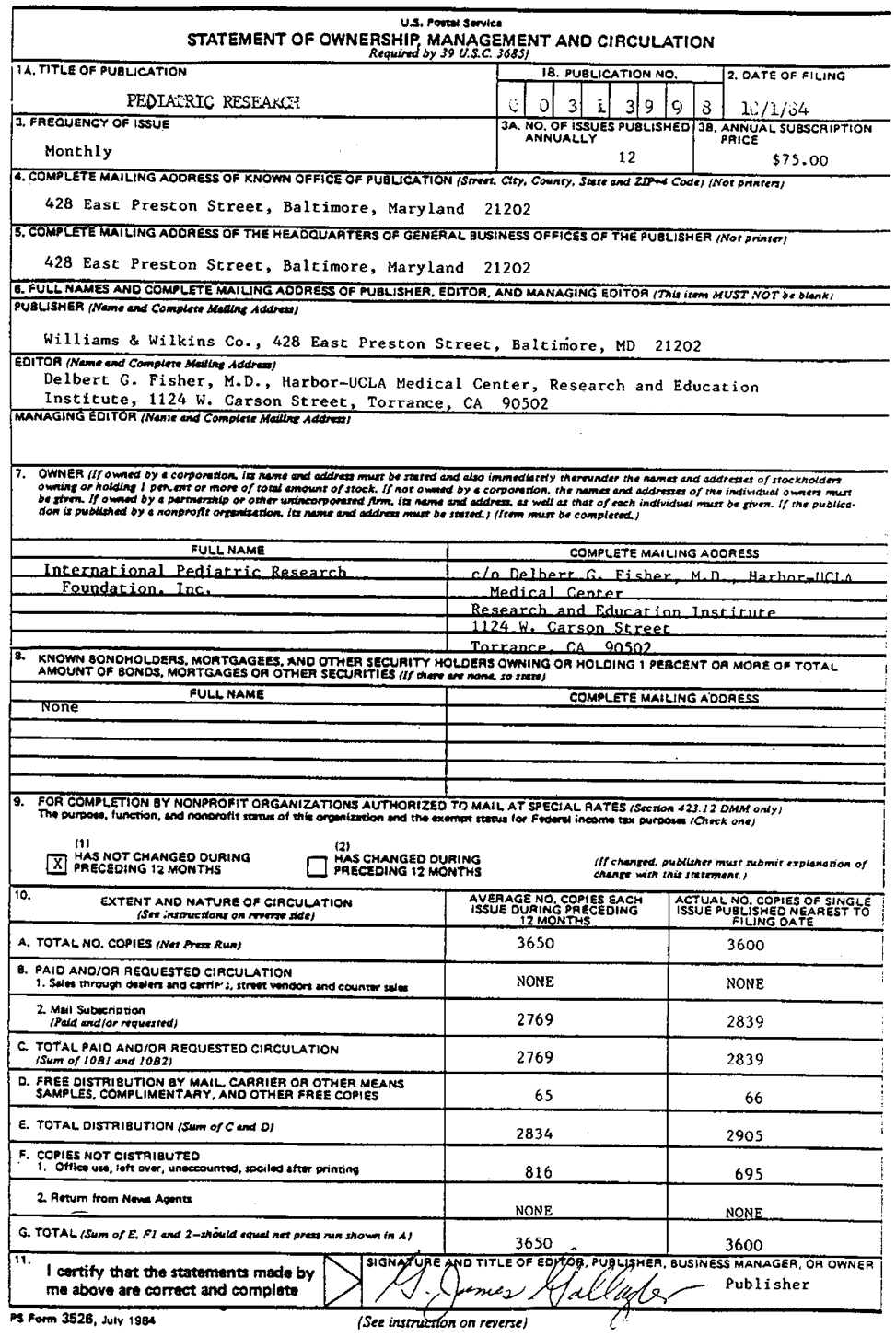

